\title{
Micro-Positron Emission Tomography Imaging of Rat Brain Metabolism during Expression of Contextual Conditioning
}

\author{
Laura Luyten, ${ }^{1,2}$ Cindy Casteels, ${ }^{3}$ Debora Vansteenwegen, ${ }^{2}$ Kris van Kuyck, ${ }^{1}$ Michel Koole, ${ }^{3}$ Koen Van Laere, ${ }^{3}$ \\ and Bart Nuttin ${ }^{1,4}$ \\ ${ }^{1}$ Division of Experimental Neurosurgery and Neuroanatomy, ${ }^{2}$ Centre for the Psychology of Learning and Experimental Psychopathology, Katholieke \\ Universiteit Leuven, 3000 Leuven, Belgium, and ${ }^{3}$ Nuclear Medicine and ${ }^{4}$ Neurosurgery, University Hospital Leuven, 3000 Leuven, Belgium
}

\begin{abstract}
Using ${ }^{18}$ F-fluorodeoxygluose microPET imaging, we investigated the neurocircuitry of contextual anxiety versus control in awake, conditioned rats ( $n=7-10$ per group). In addition, we imaged a group expressing cued fear. Simultaneous measurements of startle amplitude and freezing time were used to assess conditioning. To the best of our knowledge, no neuroimaging studies in conditioned rats have been conducted thus far, although visualizing and quantifying the metabolism of the intact brain in behaving animals is clearly of interest. In addition, more insight into the neurocircuitry involved in contextual anxiety may stimulate the development of new treatments for anxiety disorders. Our main finding was hypermetabolism in a cluster comprising the bed nucleus of the stria terminalis (BST) in rats expressing contextual anxiety compared with controls. Analysis of a subset of rats showing the best behavioral results $(n=5$ per subgroup) confirmed this finding. We also observed hypermetabolism in the same cluster in rats expressing contextual anxiety compared with rats expressing cued fear. Our results provide novel evidence for a role of the BST in the expression of contextual anxiety.
\end{abstract}

\section{Introduction}

Cued fear conditioning is obtained by repeatedly pairing an explicit cue with a shock and is regarded as a model of stimulusbound fear. Conversely, unpredictable (i.e., unsignaled) shocks produce long-term anxiety-like symptoms in the experimental context in which the shocks were previously administered. This learned response to the experimental environment is referred to as contextual conditioning and is thought to model the diffuse, generalized distress that characterizes anxiety (Davis, 1998; Fanselow, 2000; Grillon et al., 2006). To date, less is known about contextual conditioning than about cued fear conditioning, which has been studied for almost 100 years (Watson and Rayner, 1920; Pavlov and Anrep, 1927). However, interest is growing, in part because contextual conditioning in rats serves as an animal model for human pathological anxiety such as in generalized anxiety disorder (Grillon et al., 2006; Zanoveli et al., 2007; Luyten et al., 2011b). So far, the neurocircuitry of conditioning has been studied primarily through lesion or inactivation experiments in rodents (often pretraining interventions) (Kim and Jung, 2006; Walker et al., 2009) and, to a lesser degree, using autoradiography or histochemistry techniques (Gonzalez-Lima and Scheich, 1986;

\footnotetext{
Received July 20, 2011; revised 0ct. 13, 2011; accepted 0ct. 27, 2011.

Author contributions: L.L., D.V., K.v.K., and B.N. designed research; L.L. performed research; L.L., C.C., and M.K. analyzed data; L.L., C.C., D.V., K.v.K., M.K., K.V.L., and B.N. wrote the paper.

This work was supported by Research Foundation-Flanders (FW0) Project G.0729.09N and the Katholieke Universiteit Leuven Research Fund. L.L. is a doctoral fellow, C.C. and K.v.K. are postdoctoral fellows, and K.V.L. is a senior clinical investigator of the FWO. We thank Ann Van Santvoort and Peter Vermaelen for their technical support and Dr. Bridget McConnell for proofreading this manuscript. Part of these data has been presented at the Society for Neuroscience Annual Meeting (San Diego, 2010).

Correspondence should be addressed to Laura Luyten, Katholieke Universiteit Leuven, Tiensestraat 102 bus 3712 3000 Leuven, Belgium. E-mail: laura.luyten@med.kuleuven.be.

DOI:10.1523/JNEUROSCI.3701-11.2012

Copyright $\odot 2012$ the authors $\quad 0270-6474 / 12 / 320254-10 \$ 15.00 / 0$
}

Beck and Fibiger, 1995; Poremba et al., 1998; Plakke et al., 2009). To our knowledge, there are no published neuroimaging studies of conditioned rats, despite the obvious advantages of visualizing and quantifying metabolism of the intact brain in behaving animals.

In this study, we performed ${ }^{18} \mathrm{~F}$-fluorodeoxyglucose (FDG) microPET and MR imaging to study the regional brain metabolism in three groups (ANX, FEAR, and CTRL). ANX rats experienced unpredictable shocks, which were intended to produce contextual anxiety. FEAR rats received shocks signaled by a $10 \mathrm{~s}$ tone, to produce cued fear, and CTRL rats received equivalent context and tone exposure but no shocks. Contextual anxiety and cued fear were indexed by freezing and startle amplitude (Grillon, 2002; Luyten et al., 2011a). Immediately before the start of the 23 min behavioral test session, which was identical for all rats, ${ }^{18} \mathrm{~F}$-fluorodeoxyglucose was injected to ensure maximal tracer uptake during the expression of contextual anxiety or cued fear. MicroPET acquisitions were performed $1 \mathrm{~h}$ after injection during $30 \mathrm{~min}$.

We were primarily interested in the comparison between ANX and CTRL rats, to gain more insight into the neurocircuitry of contextual conditioning and because of the potential relevance of these findings to anxiety disorders (Luyten et al., $2011 b)$. We hope that, in the long run, our results will stimulate the development of new treatment options for patients suffering from anxiety disorders. Ideally, we would like to develop therapies "to reduce anxiety without preventing the treated patient from jumping out of the way of an oncoming car" (Davis et al., 1997). Therefore, we also compared the ANX with a FEAR group, to see whether we could find any neurobiological differences between animals expressing contextual anxiety and cued fear. 


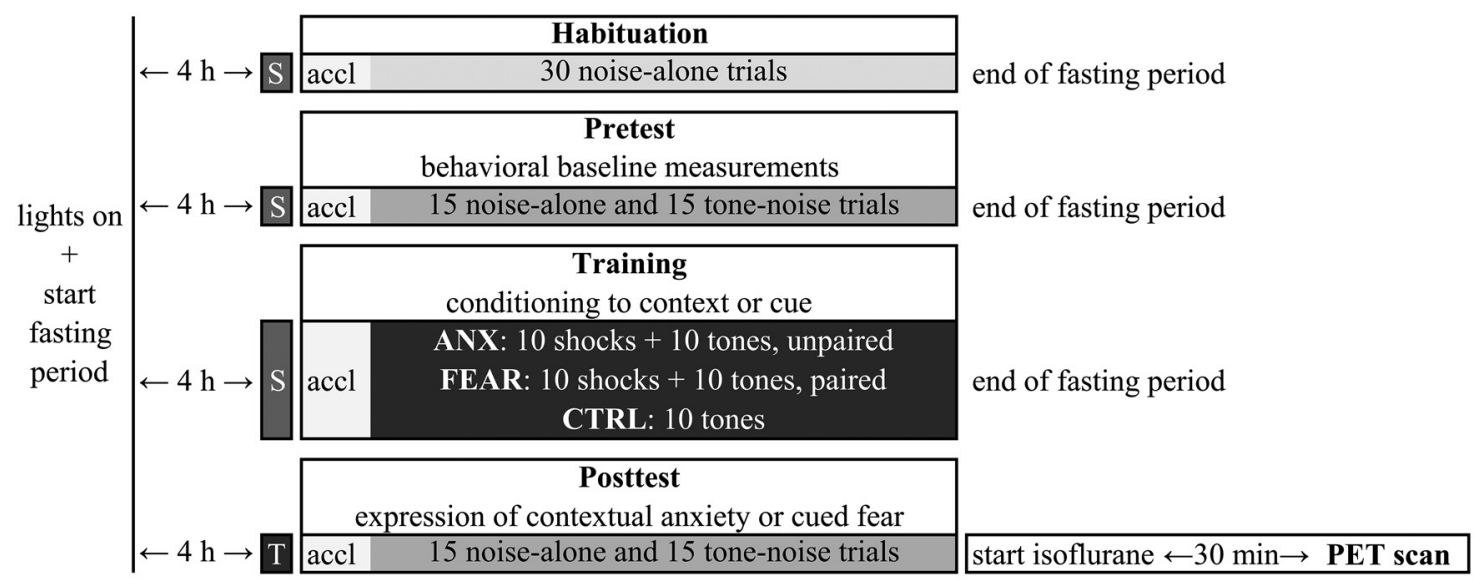

$\mathrm{S}$ saline injection, IP

T ${ }^{18}$ F-FDG injection, IP

accl $=$ acclimation

Figure 1. Experimental design.

\section{Materials and Methods \\ Subjects}

Thirty male Wistar rats (275-320 g on the habituation day) were housed separately with food and water ad libitum available. They were maintained on a $12 \mathrm{~h}$ light/dark cycle (lights on at 7:00 A.M. before the start of the experiment), with a room temperature of $\pm 22^{\circ} \mathrm{C}$. The experimental protocol was approved by the animal ethics committee of the Katholieke Universiteit Leuven, in accordance with the European Communities Council Directive of 24 November 1986 (86/609/EEC).

\section{Behavioral measurements Equipment}

During the experimental sessions, the rats were placed into a small animal cage (inner dimensions: $9.4 \mathrm{~cm}$ height, $8.2 \mathrm{~cm}$ width, and $16.5 \mathrm{~cm}$ length) with a grid floor, fixed on a response platform and located inside a ventilated sound-attenuating chamber (MED Associates). The grid floor consisted of six 5-mm-diameter stainless-steel bars spaced $10 \mathrm{~mm}$ apart, through which footshocks could be delivered (MED Associates). A red light bulb $(3.8 \mathrm{~W})$ was continuously on. The freezing behavior of the animals was recorded by a video camera (DCR-SR55E Super NightShot Plus; Sony) positioned in front of the rat holder. In addition, the startle reaction of the rats generated a pressure on the response platform and analog signals were amplified, digitized, and processed by software (Startle Reflex, version 5.95; MED Associates). The presentation and sequencing of the acoustic stimuli and footshocks were controlled by the same software. One of two loudspeakers, both located $7 \mathrm{~cm}$ behind the rat holder, was used to deliver a continuous white background noise ( $55 \mathrm{~dB}$ ); the other speaker delivered the startle (white noise, $100 \mathrm{~dB}, 50 \mathrm{~ms}$ ) and tone stimuli $(4000 \mathrm{~Hz}, 75 \mathrm{~dB}, 10 \mathrm{~s}, 5 \mathrm{~ms}$ rise/fall). The amplitude of the startle response was defined as the first peak accelerometer voltage that occurred during the first $100 \mathrm{~ms}$ after onset of the startle probe and was measured on an arbitrary scale ranging from 0 to 2047. The startle platform and loudspeakers were calibrated before the experiment.

\section{Procedure}

There were four experimental sessions separated by $24 \mathrm{~h}$ intervals: habituation, pretest, training, and posttest (Fig. 1 shows a schematic overview). Between the sessions, the animals returned to their home cages.

Habituation. On the first day, all animals were placed in the cage, and after $5 \mathrm{~min}$ of acclimation, they received 30 startle stimuli at a fixed interstimulus interval (ISI) of $30 \mathrm{~s}$. This habituation session was $20 \mathrm{~min}$ in duration and intended to habituate and stabilize the startle response (Zhao and Davis, 2004). These data were not included in further analyses.
Pretest. On the second day, a 23 min pretest was conducted to obtain behavioral baseline measurements. The animals were placed into the cage and, after 5 min of acclimation, were presented with 30 startle stimuli at a 30 s ISI. One-half of the startle stimuli were presented in the presence of a 10 s tone (i.e., 15 tone-noise trials) and the other half were presented in the absence of a tone (i.e., 15 noise-alone trials). On tonenoise trials, the startle and tone stimuli coterminated. The order of trial types was counterbalanced and randomized with the rule that no more than two trials of the same type occurred in a row. Based upon their mean startle amplitude across 15 noise-alone trials, the rats were matched into three equivalent groups (ANX, FEAR, and CTRL). A second behavioral measure assessed the time rats spent freezing during the acclimation period of $5 \mathrm{~min}$ (contextual anxiety) and during the $10 \mathrm{~s}$ preceding each startle stimulus (on noise-alone trials: measure of contextual anxiety; on tone-noise trials: measure of cued fear during the tone) as analyzed from videotapes by a blinded observer (Luyten et al., 2011a).

Training. On the third day, the rats were conditioned. The animals were placed in the box and, after 5 min of acclimation, the ANX and FEAR rats received 10 footshocks and 10 tones. The animals of the CTRL group received 10 tones, but no shocks. Each animal was removed 4 min after the last tone and returned to its home cage. The training session lasted $30 \mathrm{~min}$.

The rats in the ANX group (contextual conditioning) received explicitly unpaired presentations of the shocks $(0.8 \mathrm{~mA} ; 250 \mathrm{~ms})$ and the tones, with a variable ISI of $50-70 \mathrm{~s}$. The rats in the FEAR group (cued fear conditioning) received explicit pairings of the shocks $(0.4 \mathrm{~mA} ; 500 \mathrm{~ms})$ and tones, with a variable ISI of 100-140 s. In this group, the shocks and $10 \mathrm{~s}$ tones coterminated. The rats of the CTRL group (no conditioning) received 10 tones, with a variable ISI of $100-140 \mathrm{~s}$.

Based on preliminary data, we chose shock parameters that maximized contextual (ANX) and cued fear (FEAR) conditioning and produced a clear behavioral distinction between ANX, FEAR, and CTRL groups.

Posttest. Immediately before the start of this session, the radioactive tracer $\left({ }^{18} \mathrm{~F}\right.$-fluorodeoxyglucose $)$ was injected. Next, the animals were tested for expression of contextual anxiety or cued fear. As an additional measure, the exploratory behavior of the rats during the acclimation phase was timed. The posttest was conducted in the absence of footshocks in the same manner as the pretest. At the end of the posttest session, the rat was placed back in its home cage, transported to the adjacent scanner room, and anesthetized with isoflurane. Thirty minutes later, the microPET scan started. After the scan, the rats again received food. On the next day, structural MR images were made. 
Table 1. Inclusion criteria for subset

\begin{tabular}{llll}
\hline Group & $\begin{array}{l}\text { \% Freezing during } \\
\text { acclimation }\end{array}$ & $\begin{array}{l}\text { \% Freezing before probes, } \\
\text { difference post-pre }\end{array}$ & $\begin{array}{l}\text { Startle amplitude, } \\
\text { difference post-pre }\end{array}$ \\
\hline CTRL & $\begin{array}{l}\text { Pretest } \leq 25 \% \\
\text { Difference post-pre } \leq 0 \%\end{array}$ & $\begin{array}{l}\text { Noise alone } \leq 0 \% \\
\text { Tone-noise } \leq 0 \%\end{array}$ & $\begin{array}{l}\text { Noise alone } \leq 50 \\
\text { Tone-noise } \leq 50 \\
\text { Noise alone } \geq 10 \%\end{array}$ \\
Pretest $\leq 25 \%$ & Noise alone $\geq 50$ \\
FEAR & $\begin{array}{l}\text { Difference post-pre } \geq 10 \% \\
\text { Pretest } \leq 25 \%\end{array}$ & Tone-noise $\geq 10 \%$ & Tone-noise $\geq 50$ \\
& Difference post-pre $\leq 25 \%$ & & \\
\hline
\end{tabular}

Additional remarks. Individual light/dark cycles with food deprivation and saline injections before the sessions were added to the protocol to habituate the rat to tracer injection and fasting on the posttest day (Fig. 1).

Each rat was maintained on an individual $12 \mathrm{~h}$ light/dark cycle (lights on at 8:40, 9:20, 10:00, 10:40, 11:20 A.M., or 12:00 P.M.; the study was performed in five subsequent series of six rats each), starting $3 \mathrm{~d}$ before the habituation session. Three days should suffice to adapt to this new light/dark cycle (Fillenz and O'Neill, 1986; Miki and Sudo, 1996).

On the experimental days, food was removed at the beginning of the light period. Four hours later, an intraperitoneal injection $(0.5 \mathrm{ml}$ of saline on habituation, pretest, and training days and radioactive tracer on posttest day) was given, and a few minutes later, the experimental session started. After the session, food was returned. The fasting period preceding tracer injection ensured that the injected ${ }^{18} \mathrm{~F}$-fluorodeoxyglucose did not have to compete with high levels of normal blood glucose (Bailey, 2005; Casteels et al., 2006). For conditioning experiments, overnight fasting is not desirable because it may affect expression of contextual anxiety (L. Luyten, D. Vansteenwegen, K. van Kuyck, B. Nuttin, unpublished results). Therefore, in our protocol, food deprivation was limited to the first $4.5 \mathrm{~h}$ of the light phase, a period that was chosen to minimize the behavioral effects of fasting, since rats only consume $\sim 15 \%$ of their daily food intake during the light phase (Tempel et al., 1989; Lecklin et al., 1998).

\section{Subset selection}

Five rats were excluded from all analyses because of outlying startle amplitudes on noise-alone trials during the pretest (one rat), hematuria and allodynia (one rat), or technical problems with the image acquisition (three rats). This left 7 subjects in the CRTL group, 10 subjects in the ANX group, and 8 subjects in the FEAR group.

Because of considerable behavioral variability within these groups, we selected the best responders for additional behavioral and imaging analyses. When setting up the inclusion criteria for this subset (Table 1), we were blind to the individual microPET data.

We excluded all rats that displayed $>25 \%$ freezing during the acclimation phase of the pretest. We allowed some freezing because of possible aftereffects of the preceding saline injection, handling of the rat, and/or introduction into a rather unfamiliar environment.

Inclusion criteria for the CTRL group were as follows: no increase in percentage freezing during acclimation from pretest to posttest, no increase in percentage freezing before probes from pretest to posttest on noise-alone as well as tone-noise trials, and no considerable increase in startle amplitude from pretest to posttest on noise-alone as well as tonenoise trials. In our startle protocol, the cutoff value for startle amplitude was 50 (arbitrary scale) (i.e., startle amplitudes $<50$ were considered to be "noise" generated by small nonstartle movements of the rat and were therefore set at 0 ). Hence, differences of 50 and smaller are considered to be negligible.

Inclusion criteria for the ANX group were as follows: a minimum increase of $10 \%$ in percentage freezing during acclimation from pretest to posttest, a minimum increase of $10 \%$ in percentage freezing before noise-alone trials from pretest to posttest, and a minimum increase of 50 in startle amplitude from pretest to posttest on noise-alone trials.

Inclusion criteria for the FEAR group were as follows: a maximum increase of $25 \%$ in percentage freezing during acclimation from pretest to posttest. We allowed some background contextual freezing in the FEAR group because cued fear conditioning has been shown to produce some conditioned responses to the context. The subjects also had to demonstrate a minimum increase of $10 \%$ in percentage freezing during tone-noise trials from pretest to posttest and a minimum increase of 50 in startle amplitude from pretest to posttest on tone-noise trials.

After applying these selection criteria, we obtained a subset with five rats in each subgroup. This is a relatively small group size, but not uncommon for small-animal PET imaging studies (Barbarich-Marsteller et al., 2005; Higuera-Matas et al., 2008; Soto-Montenegro et al., 2009; Shih et al., 2011).

\section{Statistical analyses}

Difference scores ( posttest minus pretest) of startle response amplitudes (averaged for each animal across the entire session) on noise-alone trials and tone-noise trials ("Startle amplitude - Difference post-pre"), percentage freezing during acclimation ("Percentage Freezing during acclimation - Difference post-pre"), and percentage freezing during the $10 \mathrm{~s}$ period before the startle probes on noise-alone and tone-noise trials ("Percentage freezing before probes - Difference post-pre") are presented as means \pm SD (GraphPad Prism, version 4.03; GraphPad Software). Statistical analyses were used to assess conditioning in the different groups (Statistica 9; StatSoft).

Using an unpaired $t$ test, we compared "Percentage freezing during acclimation - Difference post-pre" in the ANX and CTRL groups. Twoway ANOVAs were conducted on both "Startle amplitude - Difference post-pre" and "Percentage freezing before probes - Difference postpre" with Group (ANX and CTRL) and Trial type (noise-alone and tonenoise) as factors. Tukey's post hoc tests were performed and only relevant differences (i.e., comparisons within groups, within sessions, and/or within trial types) are listed in the text or indicated on figures. A MannWhitney $U$ test was conducted on "Exploring during acclimation on posttest (in seconds)" to compare the ANX and CTRL groups. Significance levels were set at $p<0.05$.

Next, we restricted the analysis to a subset, including the rats with an optimal behavioral response (see above, Subset selection). A one-way ANOVA was conducted on "Percentage Freezing during acclimation Difference post-pre" with Subgroup (CTRL, ANX, and FEAR) as factor. Two-way ANOVAs were conducted on both "Startle amplitude - Difference post-pre" and "Percentage freezing before probes - Difference post-pre" with Subgroup (CTRL, ANX, and FEAR) and Trial type (noise-alone and tone-noise) as factors. Tukey's post hoc tests or planned comparisons (with Bonferroni's correction for multiple testing) were performed and only relevant differences (i.e., comparisons within subgroups, within sessions, and/or within trial types) are listed in the text or indicated on figures. A Kruskal-Wallis test with multiple comparisons was conducted on "Exploring during acclimation on posttest (in seconds)" to compare the three subgroups (CTRL, ANX, and FEAR). Significance levels were set at $p<0.05$.

\section{Functional and structural imaging}

\section{Radiotracer synthesis}

Brain glucose metabolism was assessed using FDG. FDG was prepared with an Ion Beam Applications FDG synthesis module. Approximately $18 \mathrm{MBq}(500 \mu \mathrm{Ci}$; specific activity range, $100-760 \mathrm{GBq} / \mu \mathrm{mol})$ of the radioligand was injected intraperitoneally. The tracer was administered in a sterile solution (injection volume, $500 \mu \mathrm{l}$ ) of $5 \mathrm{~mm}$ sodium acetate buffer, pH 5.5, containing 6\% of ethanol (Casteels et al., 2006).

\section{Data acquisition}

Small-animal PET imaging was performed using a lutetium oxyorthosilicate detector-based tomograph (microPET Focus 220; Siemens Medical Solutions), which has a transaxial resolution of $1.4 \mathrm{~mm}$ in full width at half-maximum. Data were acquired in a $128 \times 128 \times 95$ matrix with a pixel width of $0.475 \mathrm{~mm}$ and a slice thickness of $0.796 \mathrm{~mm}$. The coincidence window width was set at 6 ns with an energy window of 350-650 $\mathrm{keV}$. Before imaging, the rats were anesthetized with $2.5 \%$ isoflurane in $2.0 \mathrm{~L} / \mathrm{min}$ oxygen. FDG measurements were obtained during $30 \mathrm{~min}$ starting $1 \mathrm{~h}$ after injection. The acquisition timing rationale and kinetics of the radioligand in rats have been described previously (Casteels et al., 2006). Sinograms were reconstructed using filtered backprojection. No corrections were made for attenuation or scatter. 
MR images were acquired under isoflurane anesthesia with a smallanimal MR scanner operating at 9.4 tesla (horizontal bore, $20 \mathrm{~cm}$ bore size) (BioSpec 94/20 USR; Bruker) using a T2-weighted turboRARE sequence and a $256 \times 128 \times 256$ matrix with an isotropic pixel size of $0.137 \mathrm{~mm}$.

\section{Statistical analyses}

Relative regional glucose metabolism was determined by count normalizing FDG data to the whole-brain uptake. The within-subject test-retest variability of our imaging procedure is of comparable magnitude to human data (i.e., <5\%) (Casteels et al., 2006).

To optimally use all available image information without a priori knowledge, images were analyzed on a voxel-by-voxel basis using SPM2 (statistical parametric mapping). For spatial normalization, individual microPET and MR data were linearly normalized to custom-made rat brain templates in Paxinos stereotactic space. The procedure of spatial normalization of microPET and MR images and its validation have been described previously (Casteels et al., 2006). This methodology allows reporting results in coordinates directly corresponding to the Paxinos coordinate system for the rat brain (Paxinos and Watson, 2005).

SPM analysis was performed using a categorical subject design (groups: CTRL vs ANX vs FEAR) on the FDG data. Spatially normalized images were masked to remove extracerebral signal that would disrupt the global normalization. All images were smoothed with an isotropic Gaussian kernel of 1.2 $\mathrm{mm}$. FDG data were proportionally scaled with an analysis threshold of $80 \%$ to exclude white matter and ventricular activity.

To minimize false-positive findings, $T$-map data were interrogated at a peak level of $p<0.005$ (uncorrected) and extent threshold $k_{\mathrm{E}}>200$ voxels $\left(1.6 \mathrm{~mm}^{3}\right.$ ), as described previously (van Kuyck et al., 2007; Goffin et al., 2009). Only those clusters that were significant at the $p<0.05$ (corrected) level or that were both neurobiologically plausible and relevant in the light of other findings in this study were considered. MicroPET figures show the mean MR image (used for additional localization of the clusters) of all rats with an overlay of the functional microPET image.

In addition, a voxel-based correlation analysis was performed between relative FDG data and two covariates: freezing during acclimation on posttest (in seconds) and exploration time during acclimation on posttest (in seconds). As differences in behavioral measures were highly significant between groups, combining groups for correlation analysis on these measures would only reflect group differences. We therefore performed correlation analyses separately for the ANX group.

\section{Results}

\section{Rats expressing contextual anxiety versus controls}

\section{Comparison of ANX and CTRL groups: behavioral data}

The $t$ test conducted on startle amplitude (noise-alone trials) during pretest showed no differences between the ANX $(n=10)$ and CTRL $(n=7)$ groups $\left(t_{(15)}=0.23 ; p=0.82\right)$, indicating that matching was effective.

The $t$ test conducted on percentage freezing during acclimation (post-pre) showed significantly more contextual freezing in the ANX group than in the CTRL group $\left(t_{(15)}=4.14 ; p<0.001\right)$ (Fig. 2a). In addition, the ANOVA conducted on startle amplitude (post-pre) showed a main effect of Group $\left(F_{(1,15)}=13.75\right.$; $p<0.01$ ). Tukey's post hoc tests (Fig. $2 b$ ) showed significantly more startle potentiation in the ANX group compared with the CTRL group on both noise-alone $(p=0.03)$ and tone-noise trials $(p<0.01)$. There was no difference between startle potentiation on noise-alone and tone-noise trials within the ANX group $(p=0.40)$, suggesting that the ANX rats regarded the tones as part of the context and not as a specific fear-eliciting cue.

Finally, the ANOVA conducted on percentage freezing before probes (post-pre) showed a main effect of $\operatorname{Group}\left(F_{(1,15)}=50.55\right.$; $p<0.0001$ ). Tukey's post hoc tests (Fig. $2 c$ ) showed significantly more freezing before both noise-alone $(p<0.001)$ and tonenoise trials $(p<0.001)$ in the ANX group relative to the CTRL rats. In accordance with the startle data, there was no difference
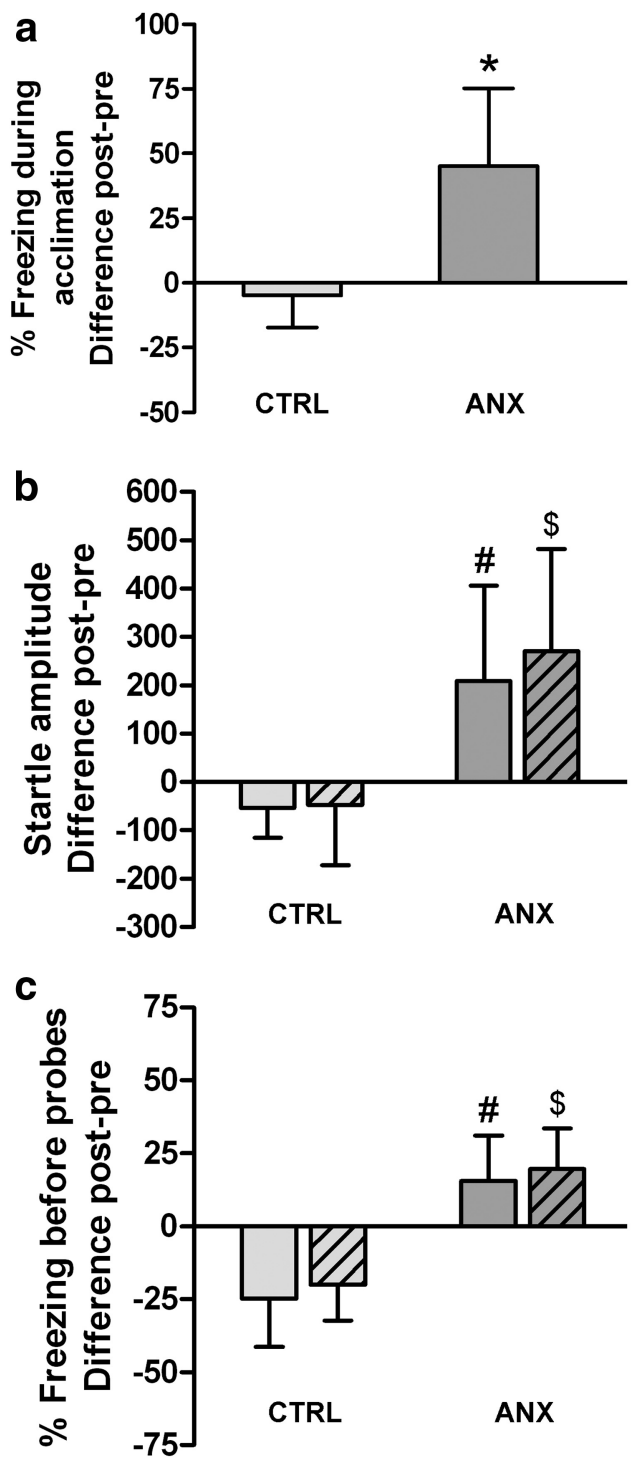

\section{$\square$ noise-alone ZZT tone-noise}

Figure 2. Effects of conditioning on percentage freezing during acclimation (a), startle amplitude $(\boldsymbol{b})$, and percentage freezing during $10 \mathrm{~s}$ before startle probes $(\boldsymbol{c})$. Difference scores of posttest minus pretest are shown (means \pm SD) for ANX $(n=10)$ and CTRL $(n=7)$ groups. *Significantly higher than CTRL ( $t$ test). "Significantly higher than CTRL noise-alone. "Significantly higher than CTRL tone-noise (ANOVA with Tukey's post hoc tests, $p<0.05$ ).

between freezing to the tone and context within the ANX group $(p=0.89)$. We can conclude that there was significant expression of contextual anxiety in the ANX group and no sign of fear or anxiety in the CTRL group.

\section{Comparison of ANX and CTRL groups: imaging data}

Relative glucose metabolism was compared between ANX and CTRL groups (Table 2), and we found a higher metabolism in the ANX group in a cluster comprising the left bed nucleus of the stria terminalis (BST), left nucleus accumbens, septal nucleus, and preoptic area. The ANX group also showed a higher metabolism in a cluster covering cingulate area 1 and 2, and in a cluster covering the dorsolateral part of the right entorhinal cortex.

Comparison with the CTRL group showed a lower metabolism in the ANX group in clusters comprising the amygdala 
Table 2. Brain regions showing glucose metabolism differences when comparing ANX ( $n=10)$ and CTRL $(n=7)$ groups and correlations between glucose metabolism and behavioral measures

\begin{tabular}{|c|c|c|c|c|c|c|c|}
\hline \multicolumn{2}{|l|}{ Cluster level } & \multicolumn{2}{|c|}{ Voxel level } & \multicolumn{3}{|c|}{ Coordinates of peak voxel } & \multirow[b]{2}{*}{ Cluster comprising } \\
\hline$p_{\text {corr }}$ & $k_{\mathrm{E}}$ & $T$ & $p_{\text {uncorr }}$ & $x$ & $y$ & $Z$ & \\
\hline \multicolumn{8}{|l|}{ ANX $>C$ TRL } \\
\hline 0.238 & 835 & 4.11 & $<0.001$ & 0.0 & 0.6 & -7.8 & $\begin{array}{l}\text { L bed nucleus of the stria terminalis (medial division, anterior and ventral part), L nucleus } \\
\text { accumbens (core and shell), medial septal nucleus and medial and lateral preoptic area }\end{array}$ \\
\hline 0.536 & 498 & 3.59 & 0.001 & -0.2 & 0.0 & -2.2 & Cingulate area 1 and 2 \\
\hline 0.871 & 238 & 3.49 & 0.001 & 7.0 & -7.2 & -7.4 & R entorhinal cortex (dorsolateral part) \\
\hline \multicolumn{8}{|l|}{ ANX $<$ CTRL } \\
\hline 0.002 & 3199 & 5.17 & $<0.001$ & 5.0 & -1.0 & -9.2 & $\begin{array}{l}\text { R amygdala (basolateral, basomedial, and lateral nuclei), } R \text { piriform cortex and } R \text { endopiriform } \\
\text { nucleus, R primary somatosensory cortex (mainly barrel field and upper lip region) }\end{array}$ \\
\hline 0.101 & 1185 & 3.89 & $<0.001$ & -4.6 & -1.6 & -9.4 & $\begin{array}{l}\text { L amygdala (basolateral, basomedial, and lateral nuclei), L piriform cortex and L endopiriform } \\
\text { nucleus }\end{array}$ \\
\hline 0.036 & 1629 & 4.86 & $<0.001$ & -4.8 & -6.8 & -4.0 & $\begin{array}{l}\text { L primary somatosensory cortex (mainly barrel field and upper lip region) and L hippocampus } \\
\text { (field CA1 and CA2) }\end{array}$ \\
\hline 0.038 & 1603 & 4.60 & $<0.001$ & 0.6 & -9.2 & -6.8 & (Latero)dorsal tegmental nucleus, dorsal raphe nucleus (caudal part), and central gray \\
\hline \multicolumn{8}{|c|}{ Positive correlation with exploring during acclimation on posttest in the ANX group } \\
\hline$<0.001$ & 2195 & 7.81 & $<0.001$ & -5.0 & -0.6 & -5.0 & L primary somatosensory cortex (upper lip region) \\
\hline
\end{tabular}

The $x$-coordinate corresponds to the distance (in millimeters) lateral to midline (right is positive), the $y$-coordinate indicates the position relative to bregma (anterior is positive), and the $z$-coordinate denotes the dorsoventral position. $p_{\text {corr }}$, cluster-corrected $p$ value; $k_{\mathrm{E}}$, cluster extent (voxels); $T$, peak voxel $t$ statistic; $p_{\text {uncorr }}$, uncorrected height $p$ value. All (de)activated brain regions are bilateral, unless stated otherwise (L, left; $R$, right).

(basolateral, basomedial, and lateral nuclei), piriform cortex, endopiriform nucleus, primary somatosensory cortex, a small portion of the left hippocampus and in a cluster comprising the (latero)dorsal tegmental nucleus, dorsal raphe nucleus, and central gray. An additional behavioral measure (exploration time during acclimation on posttest) was performed because of the metabolic changes in the somatosensory cortex. Analysis showed significantly less exploratory behavior for ANX than CTRL rats (Mann-Whitney $U$ test, $p<0.001$ ). In the ANX group, a significant positive correlation was found between the brain metabolism in the left primary somatosensory cortex and the time of exploration during the acclimation phase of the posttest.

Rats expressing contextual anxiety versus controls and versus rats expressing cued fear: a subset analysis

Inspection of the individual behavioral data showed considerable variability within each group. Therefore, we selected the best responders for additional behavioral and imaging analyses $(n=5$ per subgroup), with the intention of removing "noise" from the imaging data coming from rats that did not display the desired behavioral effects. Importantly, when setting up the inclusion criteria for the subset, we were blind to the individual microPET data.

Furthermore, as already mentioned, we were also interested in the comparison of the ANX with FEAR rats because this may be clinically relevant.

\section{Comparison of ANX, FEAR, and CTRL subgroups:}

behavioral data

The ANOVA conducted on startle amplitude (noise-alone trials) during pretest showed no differences between the three subgroups $\left(F_{(2,12)}=0.26 ; p=0.78\right)$, indicating that matching was effective.

The ANOVA conducted on percentage freezing during acclimation (post-pre) showed a main effect of Subgroup $\left(F_{(2,12)}=\right.$ 10.27 ; $p<0.01$ ). Tukey's post hoc tests (Fig. $3 a$ ) showed significantly more contextual freezing in the ANX subgroup than in both CTRL $(p<0.01)$ and FEAR $(p=0.01)$ subgroups.

In addition, the ANOVA conducted on startle amplitude (post-pre) showed a main effect of Subgroup $\left(F_{(2,12)}=9.83\right.$; $p<$ 0.01 ). Tukey's post hoc tests (Fig. $3 b$ ) showed significantly more

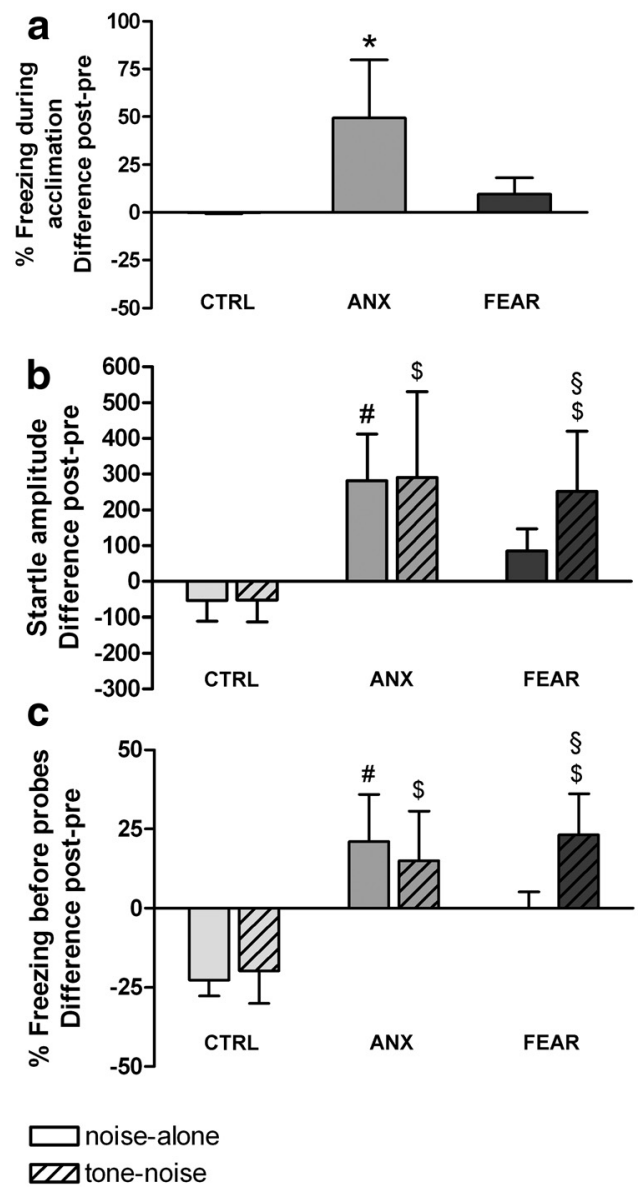

Figure 3. Effects of conditioning on percentage freezing during acclimation $(\boldsymbol{a})$, startle amplitude $(\boldsymbol{b})$, and percentage freezing during $10 \mathrm{~s}$ before startle probes $(\boldsymbol{c})$. Difference scores of posttest minus pretest of subgroups are shown (means \pm SD) $(n=5$ per subgroup). *Significantly higher than CTRL and FEAR. "Significantly higher than CTRL noise-alone. "Significantly higher than CTRL tone-noise (ANOVA with Tukey's post hoc tests, $p<0.05$ ). ${ }^{\text {SSignificantly }}$ higher than FEAR noise-alone (planned comparisons with Bonferroni's correction for multiple testing, $p<0.017)$. 
Table 3. Brain regions showing glucose metabolism differences when comparing CTRL, ANX, and FEAR subgroups ( $n=5$ per subgroup) and correlations between glucose metabolism and behavioral measures

\begin{tabular}{|c|c|c|c|c|c|c|c|}
\hline \multicolumn{2}{|l|}{ Cluster level } & \multicolumn{2}{|c|}{ Voxel level } & \multicolumn{3}{|c|}{ Coordinates of peak voxel } & \multirow[b]{2}{*}{ Cluster comprising } \\
\hline$p_{\text {corr }}$ & $k_{\mathrm{E}}$ & $T$ & $p_{\text {uncorr }}$ & $x$ & $y$ & $z$ & \\
\hline \multicolumn{8}{|l|}{ ANX $>$ CTRL } \\
\hline 0.001 & 2270 & 6.10 & $<0.001$ & 0.0 & 0.2 & -7.4 & $\begin{array}{l}\text { Bed nucleus of the stria terminalis (mainly medial and lateral division), nucleus } \\
\text { accumbens (core and shell), medial and lateral septal nucleus, and medial } \\
\text { and lateral preoptic area }\end{array}$ \\
\hline \multicolumn{8}{|l|}{ ANX $<$ CTRL } \\
\hline$<0.001$ & 3055 & 8.97 & $<0.001$ & -5.0 & -3.4 & -4.0 & $\begin{array}{l}\text { L primary somatosensory cortex (barrel field, upper lip, forelimb and hindlimb, } \\
\text { shoulder and trunk regions) and L hippocampus (field CA1 and CA2) }\end{array}$ \\
\hline$<0.001$ & 4382 & 7.86 & $<0.001$ & 5.0 & -3.4 & -4.2 & $\begin{array}{l}\text { R primary somatosensory cortex (barrel field, upper lip, forelimb and hindlimb, } \\
\text { shoulder and trunk regions) }\end{array}$ \\
\hline \multicolumn{8}{|l|}{ ANX $>$ FEAR } \\
\hline 0.301 & 577 & 4.75 & $<0.001$ & -2.0 & -1.0 & -8.6 & $\begin{array}{l}\text { Bed nucleus of the stria terminalis (mainly medial and lateral division), nucleus } \\
\text { accumbens (core and shell), medial and lateral septal nucleus, and medial } \\
\text { and lateral preoptic area }\end{array}$ \\
\hline \multicolumn{8}{|l|}{ ANX $<$ FEAR } \\
\hline 0.659 & 338 & 4.22 & 0.001 & 3.6 & -0.8 & -2.6 & R primary somatosensory cortex (mainly forelimb, shoulder, and trunk regions) \\
\hline \multicolumn{8}{|c|}{ Negative correlation with freezing during acclimation on posttest in the ANX subgroup } \\
\hline$<0.001$ & 211 & 35.87 & $<0.001$ & 5.8 & 0.6 & -3.4 & R primary somatosensory cortex (mainly upper lip region) \\
\hline \multicolumn{8}{|c|}{ Positive correlation with exploring during acclimation on posttest in the ANX subgroup } \\
\hline$<0.001$ & 265 & 34.09 & $<0.001$ & -6.0 & -1.4 & -3.6 & $\begin{array}{l}\text { L primary somatosensory cortex (mainly barrel field, upper lip, and forelimb } \\
\text { regions) }\end{array}$ \\
\hline
\end{tabular}

The $x$-coordinate corresponds to the distance (in millimeters) lateral to midline (right is positive), the $y$-coordinate indicates the position relative to bregma (anterior is positive), and the $z$-coordinate denotes the dorsoventral position. $p_{\text {corr }}$ cluster-corrected $p$ value; $k_{\mathrm{E}}$, cluster extent (voxels); $T$, peak voxel $t$ statistic; $p_{\text {uncorr }}$, uncorrected height $p$ value. All (de)activated brain regions are bilateral, unless stated otherwise (L, left; $R$, right).

startle potentiation in the ANX subgroup compared with the CTRL subgroup on both noise-alone $(p=0.01)$ and tone-noise trials $(p=0.01)$. However, there was no difference between startle potentiation on noise-alone and tone-noise trials within the ANX subgroup ( $p>0.99$ ). The FEAR subgroup showed significantly more startle potentiation on tone-noise trials than the CTRL subgroup ( $p=0.03$ ). Planned comparisons corrected for multiple testing showed significantly more startle potentiation in the FEAR subgroup on tone-noise than on noise-alone trials $(p=0.01)$.

Finally, the ANOVA conducted on percentage freezing before probes (post-pre) showed a main effect of Subgroup $\left(F_{(2,12)}=\right.$ 31.62; $p<0.0001)$ and a significant Subgroup by Trial type interaction $\left(F_{(2,12)}=4.49 ; p=0.04\right)$. Tukey's post hoc tests (Fig. $3 c$ ) showed significantly more freezing before noise-alone $(p<$ $0.001)$ and tone-noise trials $(p=0.001)$ when comparing ANX with CTRL rats. In accordance with the startle data, there was no difference in freezing to the tone or context within the ANX subgroup $(p=0.95)$. Rats in the FEAR subgroup showed more freezing before tone-noise trials than the CTRL subgroup $(p<$ 0.001). Planned comparisons corrected for multiple testing showed significantly more freezing in the FEAR subgroup before tone-noise trials than before noise-alone trials $(p<0.01)$.

Together, contextual conditioning was very low in the FEAR subgroup, while cued fear conditioning was high. However, there was no cued fear conditioning in the ANX subgroup, while contextual conditioning was high. We can conclude that there was significant expression of contextual conditioning in the ANX subgroup and cued fear conditioning in the FEAR subgroup, as evidenced by freezing and startle measurements.

Comparison of ANX and CTRL subgroups: imaging data

Relative glucose metabolism was compared between the ANX and CTRL subgroups (Table 3, Fig. 4), and the findings are in line with our observations in the full dataset. Comparison with the CTRL subgroup showed a higher metabolism in the ANX subgroup in a cluster comprising the bilateral bed nucleus of the stria terminalis, nucleus accumbens, septal nucleus, and preoptic area (Fig. 4a). Comparison with the CTRL subgroup showed a lower metabolism in the ANX subgroup in bilateral clusters comprising large parts of the primary somatosensory cortex and small portions of the hippocampus (Fig. $4 c$ ). In the ANX subgroup, a significant negative correlation was observed between freezing (during acclimation on posttest) and brain metabolism in the right primary somatosensory cortex. Additional behavioral analyses showed significantly less exploratory behavior for ANX than CTRL rats (KruskalWallis, $\mathrm{H}_{2}=9.6, p<0.01$, multiple comparisons, $\left.p<0.05\right)$. A significant positive correlation was observed between exploration time (during acclimation on posttest) and brain metabolism in the left primary somatosensory cortex.

Comparison of ANX and FEAR subgroups: imaging data

Relative glucose metabolism was compared between the ANX and FEAR subgroups (Table 3, Fig. 4). Interestingly, a comparison of the ANX and FEAR subgroups showed a hypermetabolic cluster comprising the bilateral bed nucleus of the stria terminalis, nucleus accumbens, septal nucleus, and preoptic area in the ANX rats (Fig. 4b). Although this cluster was smaller than the hypermetabolic cluster that was found when comparing ANX and CTRL subgroups (577 and 2270 voxels, respectively), the location was comparable and the brain nuclei covered by the cluster were the same.

In addition, comparison with the FEAR subgroup showed hypometabolism in the ANX rats in a cluster covering the right primary somatosensory cortex.

Finally, we did not find any hypermetabolic clusters when comparing FEAR and CTRL subgroups, but we did find two hypometabolic clusters covering parts of the primary somatosensory cortex and small portions of the left hippocampus.

\section{Discussion}

The present study investigated the neurocircuitry involved in expression of contextual anxiety (ANX) versus control (CTRL) in awake rats using metabolic microPET imaging and simultaneous 


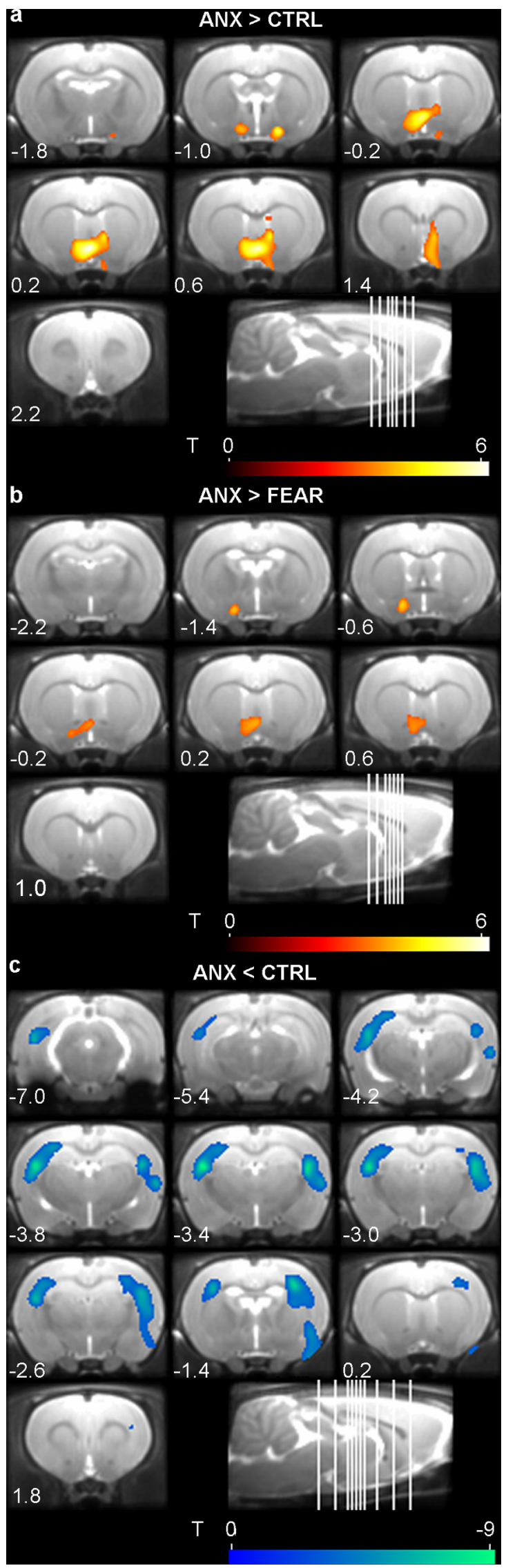

Figure 4. Mean coronal MR images of the brain showing functional microPET overlays of the clusters comprising the bed nucleus of the stria terminalis $(\boldsymbol{a}, \boldsymbol{b})$ and of the cluster covering the somatosensory cortex (c). Relative brain metabolism is increased (red) or decreased (blue) startle and freezing measurements. We also imaged a group expressing cued fear (FEAR).

Our main finding was hypermetabolism in a cluster comprising the BST in rats expressing contextual anxiety compared with controls. Analysis of a subset of rats with the best behavioral results confirmed this finding and furthermore showed hypermetabolism in the same cluster when comparing rats expressing contextual anxiety with those expressing cued fear.

A comparison of the ANX and CTRL groups showed several hypermetabolic and hypometabolic clusters.

In addition to the BST-containing cluster, which is discussed in detail below, there were also hypermetabolic clusters covering the cingulate area and parts of the right entorhinal cortex, which is consistent with previous studies that implicate the cingulate cortex in contextual conditioning (Beck and Fibiger, 1995; Pietersen et al., 2006; Skórzewska et al., 2006). In addition, the anterior cingulate cortex has been implicated in anticipatory anxiety, which may be relevant when expecting an aversive stimulus (Nitschke et al., 2006). The involvement of the entorhinal cortex in contextual conditioning is not unequivocal (Phillips and LeDoux, 1995; Schenberg et al., 2005; Majchrzak et al., 2006; Ji and Maren, 2008). Although previous data and the results from the present microPET study implicate this brain region in contextual conditioning, its exact contribution is still unclear. Studies incorporating startle as a behavioral measure may provide further elucidation.

We also observed hypometabolic clusters when comparing the ANX with CTRL group.

Our findings in the somatosensory cortex, which were confirmed in the subset analysis, support the hypothesis that increased freezing and the concomitantly decreased exploratory behavior (as seen in ANX, as opposed to the exploring CTRL rats) led to hypometabolism in this part of the cortex, covering areas involved in exploration (e.g., barrel field) (Filipkowski et al., 2000). Although this cluster also comprised small portions of the left hippocampus, we assume that this is caused by a partial volume effect, resulting in blurring of the image due to limited spatial resolution. The correlations of behavior (exploration and freezing time) with hypometabolism in the somatosensory cortex, but not in the hippocampus, support this assumption.

Other hypometabolic clusters comprised the basolateral amygdala complex (BLA) (lateral, basolateral, and basomedial nuclei of the amygdala). Through an extensive series of studies, LeDoux (2000) and Davis et al. (2010) concluded that this complex plays a crucial role in contextual and cued fear conditioning. Surprisingly, our microPET study showed that the BLA was hypometabolic in rats expressing contextual anxiety, while we were expecting an activation of this area. One possible explanation for this apparent discrepancy might be that the extensive inhibitory network of GABAergic interneurons in the BLA was deactivated (and thus hypometabolic) in the ANX rats, thereby causing an overall activation of the pyramidal projection neurons of the BLA (McDonald, 1985; Washburn and Moises, 1992; Duvarci et al., 2011). Electrophysiological recording studies in the central amygdala (another mainly GABAergic part of the amygdala) addressed this matter but yielded opposite findings

$\leftarrow$

when comparing a subset of the ANX, FEAR, and CTRL groups ( $n=5$ per subgroup) (comparison is stated on figure). Clusters are shown using a $T$ statistic color scale, which corresponds to the level of significance at the voxel level. The distance (in millimeters) relative to bregma of each section is indicated (positive values for sections anterior to bregma), as well as a sagittal view of the rat brain with depiction of the coronal slice location. Images are displayed in neurological convention, with the left side corresponding to the left hemisphere. 
including increased or decreased neuronal responses to a conditioned stimulus (Pascoe and Kapp, 1985; Ciocchi et al., 2010; Duvarci et al., 2011). Although some recording studies have been conducted in the BLA (Garcia et al., 1998; Tang et al., 2001), dedicated electrophysiological experiments should further elucidate the present findings showing a hypometabolic BLA. These future studies should take into account the presence of different cell types and focus on contextual conditioning since prior studies predominantly looked at cued fear conditioning. Additionally, they should make a clear distinction between neuronal activity during the different phases of the conditioning experiment (e.g., acquisition vs expression).

With the restriction of the analysis to a subset, we aimed to remove "noise" from the imaging data originating from rats that did not display the expected behavioral effects. It appears that this was a useful strategy. The previously found clusters covering the cingulate area, entorhinal cortex, and basolateral amygdala did not surface in the subset analysis. However, the other findings (the BST and somatosensory cortex clusters) were consistent with our observations in the full dataset. For instance, when comparing the ANX with CTRL subgroup, there was a hypermetabolic cluster covering the BST, nucleus accumbens, septal nucleus, and preoptic area. Moreover, when comparing the ANX with FEAR subgroup, we found a very similar hypermetabolic cluster. Together, this hypermetabolic BST-containing cluster seems to be a recurring observation in our study.

Note that comparison of the ANX and FEAR subgroups may have some limitations. As explained in the Introduction, we were interested in this comparison because it may be clinically relevant. Although we aimed to minimize the differences between the ANX and FEAR groups (except, of course, for the tone-shock association during training), this comparison inevitably has some limitations. In particular, because of practical issues, the ANX and FEAR rats were trained with different shock parameters and one may argue that they experienced different "exposure" times during the posttest $( \pm 23$ min exposure to the conditioned context in the ANX group and 15 times $10 \mathrm{~s}$ exposure to the conditioned tone in the FEAR group). Nevertheless, using the present parameters, the only major difference between the ANX and FEAR groups during the training session was the association between shocks and tones: explicitly unpaired in the ANX group and explicitly paired in the FEAR group. Other aspects of the experimental design, such as number of tones and shocks, interval between shocks, and length of the training session were identical for both groups. In addition, the posttest, during which there was uptake of the radioactive tracer in the brain, was identical for all rats. In summary, the ANX versus FEAR comparison is still relevant and of interest, but potential limitations should be kept in mind.

As mentioned above, the hypermetabolic BST-containing cluster in rats expressing contextual anxiety is the principal observation of this study. The BST-containing cluster also comprised parts of the nucleus accumbens, septal nucleus, and preoptic area. Both the nucleus accumbens (Westbrook et al., 1997; Pezze and Feldon, 2004; Martinez et al., 2008) and septal nucleus (Beck and Fibiger, 1995; Sparks and LeDoux, 1995; Calandreau et al., 2007; Reis et al., 2010) have at times been implicated in contextual conditioning, even though their exact roles remain unclear. The preoptic area seems to play a role in defensive and anxious behaviors (Briski and Gillen, 2001; McGregor et al., 2004; Salomé et al., 2004; Schwartz et al., 2008), but we found no studies providing direct evidence for its involvement in contextual conditioning.

However, we believe that the BST, in particular, is the pivotal brain region in the observed hypermetabolic cluster. The overall picture of the neurocircuitry of contextual conditioning is still unclear, but several brain regions, including the BST, seem to be involved. As to be expected, we did not observe metabolic changes in all of these regions. Studies using more animals may provide better power to detect additional brain areas. Also, our current study focused on the expression phase, as this is the most relevant phase regarding anxiety disorders. At present, it is not always clear whether the brain regions in the proposed neurocircuitries play a role in, for example, acquisition or expression.

Walker et al. (2003) and Davis et al. (2010) have primarily investigated the involvement of the amygdala and BST and the anatomical dissociation between contextual anxiety and cued fear, or as they prefer to call it, sustained and phasic fear. They state that sensory information (e.g., coming from a threatening context) enters the BLA, which projects to the BST and also sends light projections to the lateral division of the central amygdala, which in turn projects heavily and more slowly to the BST. Finally, the BST projects to various brain regions involved in the behavioral and autonomic consequences of anxiety [e.g., periaqueductal gray (freezing) and caudal pontine reticular nucleus (startle potentiation)] (Walker et al., 2003).

Other groups have focused on the role of the hippocampus in contextual conditioning. They suggest that the hippocampus provides input about complex, multimodal stimuli, such as the experimental context in contextual conditioning. This is in line with the generally acknowledged role of the hippocampus in spatial learning (Phillips and LeDoux, 1992; Gewirtz et al., 2000). Note that the hippocampus has mainly been implicated in the acquisition of contextual conditioning (Kim and Fanselow, 1992; Phillips and LeDoux, 1992; Anagnostaras et al., 2001; Marschner et al., 2008). This might explain why we did not observe any distinct metabolic changes in the hippocampus in the present study investigating the expression phase.

Finally, researchers are starting to unveil other brain areas that might be involved. Using FDG-PET in monkeys, it was found that the orbitofrontal cortex has modulatory inputs into the BST and may play a role in the adaptive regulation of anxiety responses (Fox et al., 2010).

Involvement of the BST in anxiety is supported by a growing body of preclinical evidence (for an overview, see Walker et al., 2009; Davis et al., 2010). It has been demonstrated that BST lesions disrupt contextual freezing in a previously shocked context (Onaka and Yagi, 1998; Nijsen et al., 2001; Sullivan et al., 2004; Resstel et al., 2008; Zimmerman and Maren, 2011), but not freezing to a conditioned cue nor fear-potentiated startle following cued fear conditioning (LeDoux et al., 1988; Walker et al., 2003). Very recently, we found that posttraining BST lesions completely disrupted the expression of contextual anxiety, as measured simultaneously with startle amplitude and freezing, in rats after reintroduction in a previously shocked context (Luyten et al., 2011c). The stereotactic coordinates for the location of the BST lesions were calculated based upon the present microPET findings. Since the electrolytic lesion was principally restricted to the BST, these results support our hypothesis that it is indeed the BST that is hypermetabolic and essential in the present study. The resolution of microPET imaging (which is in the order of magnitude of the size of the BST itself) may be a limitation of the present study, and the hypermetabolic cluster including the BST also comprised parts of other brain regions. Strictly speaking, one could argue that the hypermetabolism in the BST was just a consequence of the partial volume effect of surrounding hypermetabolic brain regions. However, the results of our lesion study corroborate that the BST plays a key role in the expression of contextual anxiety. 
Since a few years, imaging studies are beginning to provide additional support for a role of the BST in anxiety. An FDG-PET imaging study in rhesus monkeys found a correlation between BST metabolism and freezing in a human intruder paradigm or after separation from cage mates (Kalin et al., 2005). A functional MRI (fMRI) study in spider phobics found increased activation in the left BST during anticipation of spider versus neutral pictures (Straube et al., 2007). An fMRI study in healthy participants concluded that the BST indexes hypervigilant threat monitoring (Somerville et al., 2010). Another fMRI study in healthy participants found BST activity when comparing unpredictable threat with no threat using virtual reality contexts (Alvarez et al., 2011).

Anxiety, as opposed to stimulus-bound fear, is also an aspect of many anxiety and other psychiatric disorders. It is noteworthy that we found that deep brain stimulation of the BST alleviates symptoms in patients suffering from severe obsessive-compulsive disorder, a debilitating anxiety disorder (Gabriëls et al., 2007; Greenberg et al., 2010). Accordingly, we showed that high-frequency electrical stimulation of the BST in a rat model for obsessive-compulsive disorder decreased compulsive behavior (van Kuyck et al., 2008).

To conclude, the present study clearly supports a key role for the BST in the expression of contextual anxiety, which is an animal model for anxiety-like behavior. Thereby it provides novel evidence for a role for the BST as a promising target, be it pharmacological or neurosurgical, in the future treatment of pathological anxiety.

\section{References}

Alvarez RP, Chen G, Bodurka J, Kaplan R, Grillon C (2011) Phasic and sustained fear in humans elicits distinct patterns of brain activity. Neuroimage 55:389-400.

Anagnostaras SG, Gale GD, Fanselow MS (2001) Hippocampus and contextual fear conditioning: recent controversies and advances. Hippocampus 11:8-17.

Bailey DL (2005) Positron emission tomography: basic sciences. London: Springer.

Barbarich-Marsteller NC, Marsteller DA, Alexoff DL, Fowler JS, Dewey SL (2005) MicroPET imaging in an animal model of anorexia nervosa. Synapse 57:85-90.

Beck CH, Fibiger HC (1995) Conditioned fear-induced changes in behavior and in the expression of the immediate early gene c-fos: with and without diazepam pretreatment. J Neurosci 15:709-720.

Briski K, Gillen E (2001) Differential distribution of Fos expression within the male rat preoptic area and hypothalamus in response to physical vs. psychological stress. Brain Res Bull 55:401-408.

Calandreau L, Jaffard R, Desmedt A (2007) Dissociated roles for the lateral and medial septum in elemental and contextual fear conditioning. Learn Mem 14:422-429.

Casteels C, Vermaelen P, Nuyts J, Van Der Linden A, Baekelandt V, Mortelmans L, Bormans G, Van Laere K (2006) Construction and evaluation of multitracer small-animal PET probabilistic atlases for voxel-based functional mapping of the rat brain. J Nucl Med 47:1858-1866.

Ciocchi S, Herry C, Grenier F, Wolff SB, Letzkus JJ, Vlachos I, Ehrlich I, Sprengel R, Deisseroth K, Stadler MB, Müller C, Lüthi A (2010) Encoding of conditioned fear in central amygdala inhibitory circuits. Nature 468:277-282.

Davis M (1998) Are different parts of the extended amygdala involved in fear versus anxiety? Biol Psychiatry 44:1239-1247.

Davis M, Walker DL, Lee Y (1997) Amygdala and bed nucleus of the stria terminalis: differential roles in fear and anxiety measured with the acoustic startle reflex. Philos Trans R Soc Lond B Biol Sci 352:1675-1687.

Davis M, Walker DL, Miles L, Grillon C (2010) Phasic vs sustained fear in rats and humans: role of the extended amygdala in fear vs anxiety. Neuropsychopharmacology 35:105-135.

Duvarci S, Popa D, Paré D (2011) Central amygdala activity during fear conditioning. J Neurosci 31:289-294.

Fanselow MS (2000) Contextual fear, gestalt memories, and the hippocampus. Behav Brain Res 110:73-81.
Filipkowski RK, Rydz M, Berdel B, Morys J, Kaczmarek L (2000) Tactile experience induces c-fos expression in rat barrel cortex. Learn Mem $7: 116-122$.

Fillenz M, O'Neill RD (1986) Effects of light reversal on the circadian pattern of motor activity and voltammetric signals recorded in rat forebrain. J Physiol 374:91-101.

Fox AS, Shelton SE, Oakes TR, Converse AK, Davidson RJ, Kalin NH (2010) Orbitofrontal cortex lesions alter anxiety-related activity in the primate bed nucleus of stria terminalis. J Neurosci 30:7023-7027.

Gabriëls L, Nuttin B, Cosyns P (2007) DBS and the treatment of obsessive compulsive disorder. In: Proceedings of the Medtronic Forum for Neuroscience and Neuro-Technology 2005, pp 57-61. Berlin, Heidelberg: Springer.

Garcia R, Paquereau J, Vouimba RM, Jaffard R (1998) Footshock stress but not contextual fear conditioning induces long-term enhancement of auditory-evoked potentials in the basolateral amygdala of the freely behaving rat. Eur J Neurosci 10:457-463.

Gewirtz JC, McNish KA, Davis M (2000) Is the hippocampus necessary for contextual fear conditioning? Behav Brain Res 110:83-95.

Goffin K, Van Paesschen W, Dupont P, Van Laere K (2009) Longitudinal microPET imaging of brain glucose metabolism in rat lithiumpilocarpine model of epilepsy. Exp Neurol 217:205-209.

Gonzalez-Lima F, Scheich H (1986) Classical conditioning of tone-signaled bradycardia modifies 2-deoxyglucose uptake patterns in cortex, thalamus, habenula, caudate-putamen and hippocampal formation. Brain Res 363:239-256.

Greenberg BD, Gabriels LA, Malone DA Jr, Rezai AR, Friehs GM, Okun MS, Shapira NA, Foote KD, Cosyns PR, Kubu CS, Malloy PF, Salloway SP, Giftakis JE, Rise MT, Machado AG, Baker KB, Stypulkowski PH, Goodman WK, Rasmussen SA, Nuttin BJ (2010) Deep brain stimulation of the ventral internal capsule/ventral striatum for obsessive-compulsive disorder: worldwide experience. Mol Psychiatry 15:64-79.

Grillon C (2002) Startle reactivity and anxiety disorders: aversive conditioning, context, and neurobiology. Biol Psychiatry 52:958-975.

Grillon C, Baas JM, Cornwell B, Johnson L (2006) Context conditioning and behavioral avoidance in a virtual reality environment: effect of predictability. Biol Psychiatry 60:752-759.

Higuera-Matas A, Soto-Montenegro ML, del Olmo N, Miguéns M, Torres I, Vaquero JJ, Sánchez J, García-Lecumberri C, Desco M, Ambrosio E (2008) Augmented acquisition of cocaine self-administration and altered brain glucose metabolism in adult female but not male rats exposed to a cannabinoid agonist during adolescence. Neuropsychopharmacology 33:806-813.

Ji J, Maren S (2008) Lesions of the entorhinal cortex or fornix disrupt the context-dependence of fear extinction in rats. Behav Brain Res 194:201-206.

Kalin NH, Shelton SE, Fox AS, Oakes TR, Davidson RJ (2005) Brain regions associated with the expression and contextual regulation of anxiety in primates. Biol Psychiatry 58:796-804.

Kim JJ, Fanselow MS (1992) Modality-specific retrograde amnesia of fear. Science 256:675-677.

Kim JJ, Jung MW (2006) Neural circuits and mechanisms involved in Pavlovian fear conditioning: a critical review. Neurosci Biobehav Rev 30:188-202.

Lecklin A, Etu-Seppälä P, Stark H, Tuomisto L (1998) Effects of intracerebroventricularly infused histamine and selective $\mathrm{H} 1, \mathrm{H} 2$ and $\mathrm{H} 3$ agonists on food and water intake and urine flow in Wistar rats. Brain Res 793:279-288.

LeDoux JE (2000) Emotion circuits in the brain. Annu Rev Neurosci 23:155-184

LeDoux JE, Iwata J, Cicchetti P, Reis DJ (1988) Different projections of the central amygdaloid nucleus mediate autonomic and behavioral correlates of conditioned fear. J Neurosci 8:2517-2529.

Luyten L, Vansteenwegen D, van Kuyck K, Deckers D, Nuttin B (2011a) Optimization of a contextual conditioning protocol for rats using combined measurements of startle amplitude and freezing: the effects of shock intensity and different types of conditioning. J Neurosci Methods 194:305-311.

Luyten L, Vansteenwegen D, van Kuyck K, Gabriëls L, Nuttin B (2011b) Contextual conditioning in rats as an animal model for generalized anxiety disorder. Cogn Affect Behav Neurosci 11:228-244.

Luyten L, van Kuyck K, Vansteenwegen D, Nuttin B (2011c) Electrolytic 
lesions of the bed nucleus of the stria terminalis disrupt freezing and startle potentiation in a conditioned context. Behav Brain Res 222:357-362.

Majchrzak M, Ferry B, Marchand AR, Herbeaux K, Seillier A, Barbelivien A (2006) Entorhinal cortex lesions disrupt fear conditioning to background context but spare fear conditioning to a tone in the rat. Hippocampus 16:114-124.

Marschner A, Kalisch R, Vervliet B, Vansteenwegen D, Büchel C (2008) Dissociable roles for the hippocampus and the amygdala in human cued versus context fear conditioning. J Neurosci 28:9030-9036.

Martinez RC, Oliveira AR, Macedo CE, Molina VA, Brandão ML (2008) Involvement of dopaminergic mechanisms in the nucleus accumbens core and shell subregions in the expression of fear conditioning. Neurosci Lett 446:112-116.

McDonald AJ (1985) Immunohistochemical identification of gammaaminobutyric acid-containing neurons in the rat basolateral amygdala. Neurosci Lett 53:203-207.

McGregor IS, Hargreaves GA, Apfelbach R, Hunt GE (2004) Neural correlates of cat odor-induced anxiety in rats: region-specific effects of the benzodiazepine midazolam. J Neurosci 24:4134-4144.

Miki K, Sudo A (1996) Adaptation of circadian corticosterone and catecholamine rhythms to light-dark cycle reversal in the rat. Ind Health 34:133-138.

Nijsen MJ, Croiset G, Diamant M, De Wied D, Wiegant VM (2001) CRH signalling in the bed nucleus of the stria terminalis is involved in stressinduced cardiac vagal activation in conscious rats. Neuropsychopharmacology 24:1-10.

Nitschke JB, Sarinopoulos I, Mackiewicz KL, Schaefer HS, Davidson RJ (2006) Functional neuroanatomy of aversion and its anticipation. Neuroimage 29:106-116.

Onaka T, Yagi K (1998) Role of noradrenergic projections to the bed nucleus of the stria terminalis in neuroendocrine and behavioral responses to fear-related stimuli in rats. Brain Res 788:287-293.

Pascoe JP, Kapp BS (1985) Electrophysiological characteristics of amygdaloid central nucleus neurons during Pavlovian fear conditioning in the rabbit. Behav Brain Res 16:117-133.

Pavlov IP, Anrep GV (1927) Conditioned reflexes; an investigation of the physiological activity of the cerebral cortex. London: Humphrey Milford, Oxford UP.

Paxinos G, Watson C (2005) The rat brain in stereotaxic coordinates, Ed 5. Amsterdam: Elsevier Academic.

Pezze MA, Feldon J (2004) Mesolimbic dopaminergic pathways in fear conditioning. Prog Neurobiol 74:301-320.

Phillips RG, LeDoux JE (1992) Differential contribution of amygdala and hippocampus to cued and contextual fear conditioning. Behav Neurosci 106:274-285.

Phillips RG, LeDoux JE (1995) Lesions of the fornix but not the entorhinal or perirhinal cortex interfere with contextual fear conditioning. J Neurosci 15:5308-5315.

Pietersen CY, Bosker FJ, Postema F, Fokkema DS, Korf J, den Boer JA (2006) Ketamine administration disturbs behavioural and distributed neural correlates of fear conditioning in the rat. Prog Neuropsychopharmacol Biol Psychiatry 30:1209-1218.

Plakke B, Freeman JH, Poremba A (2009) Metabolic mapping of rat forebrain and midbrain during delay and trace eyeblink conditioning. Neurobiol Learn Mem 92:335-344.

Poremba A, Jones D, Gonzalez-Lima F (1998) Classical conditioning modifies cytochrome oxidase activity in the auditory system. Eur J Neurosci 10:3035-3043.

Reis DG, Scopinho AA, Guimarães FS, Corrêa FM, Resstel LB (2010) Involvement of the lateral septal area in the expression of fear conditioning to context. Learn Mem 17:134-138.

Resstel LB, Alves FH, Reis DG, Crestani CC, Corrêa FM, Guimarães FS (2008) Anxiolytic-like effects induced by acute reversible inactivation of the bed nucleus of stria terminalis. Neuroscience 154:869-876.

Salomé N, Salchner P, Viltart O, Sequeira H, Wigger A, Landgraf R, Singewald N (2004) Neurobiological correlates of high (HAB) versus low anxietyrelated behavior (LAB): differential Fos expression in $\mathrm{HAB}$ and $\mathrm{LAB}$ rats. Biol Psychiatry 55:715-723.

Schenberg EE, Soares JC, Oliveira MG (2005) Effects of pre- or posttraining entorhinal cortex AP5 injection on fear conditioning. Physiol Behav 86:508-515.
Schwartz JA, Reilly NS, Knuepfer MM (2008) Angiotensin and NMDA receptors in the median preoptic nucleus mediate hemodynamic response patterns to stress. Am J Physiol Regul Integr Comp Physiol 295:R155-R165.

Shih YY, Wey HY, De La Garza BH, Duong TQ (2011) Striatal and cortical BOLD, blood flow, blood volume, oxygen consumption, and glucose consumption changes in noxious forepaw electrical stimulation. J Cereb Blood Flow Metab 31:832-841.

Skórzewska A, Bidzinski A, Lehner M, Turzynska D, Wislowska-Stanek A, Sobolewska A, Szyndler J, Maciejak P, Taracha E, Plaznik A (2006) The effects of acute and chronic administration of corticosterone on rat behavior in two models of fear responses, plasma corticosterone concentration, and c-Fos expression in the brain structures. Pharmacol Biochem Behav 85:522-534.

Somerville LH, Whalen PJ, Kelley WM (2010) Human bed nucleus of the stria terminalis indexes hypervigilant threat monitoring. Biol Psychiatry 68:416-424.

Soto-Montenegro ML, Vaquero JJ, Pascau J, Gispert JD, García-Barreno P, Desco M (2009) Detection of visual activation in the rat brain using 2-deoxy-2- $\left[{ }^{18} \mathrm{~F}\right]$ fluoro-D-glucose and statistical parametric mapping (SPM). Mol Imaging Biol 11:94-99.

Sparks PD, LeDoux JE (1995) Septal lesions potentiate freezing behavior to contextual but not to phasic conditioned stimuli in rats. Behav Neurosci 109:184-188.

Straube T, Mentzel HJ, Miltner WH (2007) Waiting for spiders: brain activation during anticipatory anxiety in spider phobics. Neuroimage 37:1427-1436.

Sullivan GM, Apergis J, Bush DE, Johnson LR, Hou M, Ledoux JE (2004) Lesions in the bed nucleus of the stria terminalis disrupt corticosterone and freezing responses elicited by a contextual but not by a specific cueconditioned fear stimulus. Neuroscience 128:7-14.

Tang J, Wotjak CT, Wagner S, Williams G, Schachner M, Dityatev A (2001) Potentiated amygdaloid auditory-evoked potentials and freezing behavior after fear conditioning in mice. Brain Res 919:232-241.

Tempel DL, Shor-Posner G, Dwyer D, Leibowitz SF (1989) Nocturnal patterns of macronutrient intake in freely feeding and food-deprived rats. Am J Physiol 256:R541-R548.

van Kuyck K, Casteels C, Vermaelen P, Bormans G, Nuttin B, Van Laere K (2007) Motor- and food-related metabolic cerebral changes in the activity-based rat model for anorexia nervosa: a voxel-based microPET study. Neuroimage 35:214-221.

van Kuyck K, Brak K, Das J, Rizopoulos D, Nuttin B (2008) Comparative study of the effects of electrical stimulation in the nucleus accumbens, the mediodorsal thalamic nucleus and the bed nucleus of the stria terminalis in rats with schedule-induced polydipsia. Brain Res 1201:93-99.

Walker DL, Toufexis DJ, Davis M (2003) Role of the bed nucleus of the stria terminalis versus the amygdala in fear, stress, and anxiety. Eur J Pharmacol 463:199-216.

Walker DL, Miles LA, Davis M (2009) Selective participation of the bed nucleus of the stria terminalis and CRF in sustained anxiety-like versus phasic fear-like responses. Prog Neuropsychopharmacol Biol Psychiatry 33:1291-1308.

Washburn MS, Moises HC (1992) Electrophysiological and morphological properties of rat basolateral amygdaloid neurons in vitro. J Neurosci 12:4066-4079.

Watson JB, Rayner R (1920) Conditioned emotional responses. J Exp Psychol 3:1-14

Westbrook RF, Good AJ, Kiernan MJ (1997) Microinjection of morphine into the nucleus accumbens impairs contextual learning in rats. Behav Neurosci 111:996-1013.

Zanoveli JM, Ferreira-Netto C, Brandão ML (2007) Conditioned place aversion organized in the dorsal periaqueductal gray recruits the laterodorsal nucleus of the thalamus and the basolateral amygdala. Exp Neurol 208:127-136.

Zhao Z, Davis M (2004) Fear-potentiated startle in rats is mediated by neurons in the deep layers of the superior colliculus/deep mesencephalic nucleus of the rostral midbrain through the glutamate non-NMDA receptors. J Neurosci 24:10326-10334.

Zimmerman JM, Maren S (2011) The bed nucleus of the stria terminalis is required for the expression of contextual but not auditory freezing in rats with basolateral amygdala lesions. Neurobiol Learn Mem 95: 199-205. 